\title{
Quantitative effect of CYP2D6 genotype and inhibitors on tamoxifen metabolism: Implication for optimization of breast cancer treatment
}

Background and Objectives: N-Desmethyltamoxifen (NDM), a major primary metabolite of tamoxifen, is hydroxylated by cytochrome P450 (CYP) 2D6 to yield endoxifen. Because of its high antiestrogenic potency, endoxifen may play an important role in the clinical activity of tamoxifen. We conducted a prospective trial in 158 patients with breast cancer who were taking tamoxifen to further understand the effect of CYP2D6 genotype and concomitant medications on endoxifen plasma concentrations.

Methods: Medication history, genotype for 33 CYP2D6 alleles, and plasma concentrations of tamoxifen and its metabolites were determined at the fourth month of tamoxifen treatment.

Results: By use of a mixture model approach, endoxifen plasma concentration identified 2 phenotypic groups, whereas 4 were defined by the endoxifen/NDM plasma concentration ratio. Three distinct genotype groups were identified in the distribution of endoxifen/NDM ratio: (1) low ratios composed of patients lacking any functional allele (mean, $0.04 \pm 0.02$ ); (2) intermediate ratios represented by patients with 1 active allele (mean, $0.08 \pm 0.04$ ); and (3) high ratios composed of patients with 2 or more functional alleles (mean, 0.15 $\pm 0.09)$. Endoxifen/NDM plasma ratios were significantly different between these groups $(P<.001)$. The mean endoxifen plasma concentration was significantly lower in CYP2D6 extensive metabolizers who were taking potent CYP2D6 inhibitors than in those who were not taking CYP2D6 inhibitors $(23.5 \pm 9.5$ $\mathrm{nmol} / \mathrm{L}$ versus $84.1 \pm 39.4 \mathrm{nmol} / \mathrm{L}, P<.001)$.

Conclusion: CYP2D6 genotype and concomitant potent CYP2D6 inhibitors are highly associated with endoxifen plasma concentration and may have an impact on the response to tamoxifen therapy. These iterative approaches may be valuable in the study of other complex genotype-phenotype relationships. (Clin Pharmacol Ther 2006;80:61-74.)

Silvana Borges, MD, Zeruesenay Desta, PhD, Lang Li, PhD, Todd C. Skaar, PhD, Bryan A. Ward, BSc, Anne Nguyen, BSc, Yan Jin, MD, Anna Maria Storniolo, MD, D. Michele Nikoloff, PhD, Lin Wu, PhD, Grant Hillman, MSc, Daniel F. Hayes, MD, Vered Stearns, MD, and David A. Flockhart, MD, PhD Indianapolis, Ind, Ann Arbor, Mich, Baltimore, $M d$, and Pleasanton, Calif

From the Division of Clinical Pharmacology, Department of Medicine, Indiana University School of Medicine, Indianapolis; Breast Oncology Program, University of Michigan, Ann Arbor; Breast Cancer Program, Sidney Kimmel Comprehensive Cancer Center, Johns Hopkins School of Medicine, Baltimore; and Roche Molecular Diagnostics, Pleasanton.

Drs Borges and Desta contributed equally to this work.

This study was supported in part by a Pharmacogenetics Research Network grant (2U-01 GM61373) (D.A.F., D.F.H., and V.S.), grant K24RR020815 (D.A.F.) from the National Center for Research Resources, Bethesda, Md, and a clinical pharmacology training grant (5T32-GM-08425) (D.A.F.) from the National Institute of General Medical Sciences, Bethesda, Md; by Damon
Runyon-Lilly Clinical Investigator Award CI-3 from the Damon Runyon Cancer Research Foundation (V.S.); by the Fashion Footwear Foundation/QVC Presents Shoes on Sale (D.F.H.); and by a Merck Sharp \& Dohme International Fellowship in Clinical Pharmacology (S.B.).

Reprint requests: Silvana Borges, MD, Division of Clinical Pharmacology, Indiana University School of Medicine, 1001 W 10th St, WD Myers Bldg, W7123, Indianapolis, IN 46202.

E-mail: siborges@iupui.edu

0009-9236/\$32.00

Copyright () 2006 by the American Society for Clinical Pharmacology and Therapeutics.

doi:10.1016/j.clpt.2006.03.013 
Tamoxifen, a selective estrogen receptor modulator, is commonly used for the treatment and prevention of breast cancer. ${ }^{1,2}$ The clinical response to tamoxifen varies widely among patients, ${ }^{2}$ and the identification of determinants of this variability is important, especially in an era of personalized medicine. Tamoxifen undergoes extensive hepatic and gut wall metabolism in humans to several primary and secondary metabolites that exhibit a range of pharmacologic activity. ${ }^{3,4}$ Therefore differences in systemic exposure of one or more of its active metabolites likely contribute to the variable response of tamoxifen observed in patients with breast cancer. ${ }^{5}$

Since its first description in $1977,{ }^{6}$ 4-hydroxytamoxifen has been considered to be the principal active metabolite of tamoxifen because of its high affinity for estrogen receptors and 30- to 100-fold greater potency than tamoxifen in suppressing estrogen-dependent breast cancer cell proliferation in vitro. ${ }^{6-8}$ However, our group has recently investigated another metabolite of tamoxifen, 4-hydroxy- $\mathrm{N}$ desmethyltamoxifen (endoxifen) (Fig 1). Although this metabolite was reported in the 1980s in humans, ${ }^{9}$ its pharmacologic relevance remained unknown. Through a series of laboratory and clinical studies, we have demonstrated that (1) endoxifen formation proceeds stepwise by oxidation of tamoxifen with $\mathrm{N}$-desmethyltamoxifen (NDM) as the predominant intermediate (Fig 1), ${ }^{3}$ (2) endoxifen has a potency in vitro that is equivalent to the potency of 4hydroxytamoxifen and it reaches greater than 6-fold higher plasma concentrations, on average, than 4-hydroxytamoxifen in patients taking tamoxifen, ${ }^{7,10}$ and (3) plasma concentrations in patients receiving tamoxifen are influenced by cytochrome P450 (CYP) 2D6 genetic variants and concomitant intake of known CYP2D6 inhibitor drugs (eg, paroxetine). ${ }^{7,10}$

Evidence that CYP2D6 activity is a determinant of tamoxifen efficacy and adverse effects has been obtained from our recent retrospective analysis in which breast cancer patients who were poor metabolizers of CYP2D6 had a worse clinical outcome (increased recurrence and mortality rates) and fewer adverse effects compared with those who were extensive metabolizers of CYP2D6. ${ }^{11}$ It follows that an improved understanding of factors that influence CYP2D6 activity in breast cancer patients and its consequences on endoxifen formation is important to the rational optimization of tamoxifen therapy.

CYP2D6 activity is highly variable in the human population, ${ }^{12-14}$ largely as a result of polymorphisms in the $C Y P 2 D 6$ gene. ${ }^{15} C Y P 2 D 6{ }^{*} 1$ is the wild-type allele,

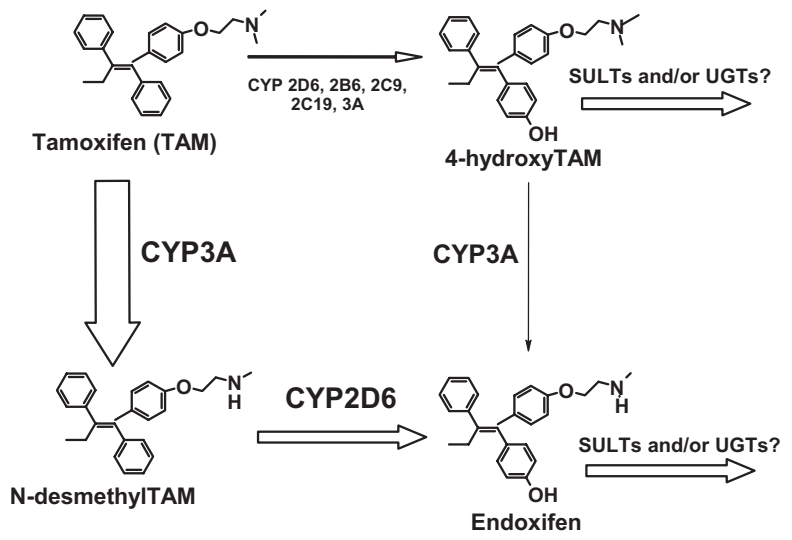

Fig 1. Sequential biotransformation of tamoxifen (TAM) to endoxifen in humans. Tamoxifen is predominantly $\mathrm{N}$-demethylated by the CYP3A enzyme to $N$-desmethyltamoxifen, which is a major primary tamoxifen metabolite quantitatively. (At steady state, the plasma concentration of this metabolite is more than 1.5-fold higher than that of tamoxifen after 20$\mathrm{mg} / \mathrm{d}$ treatment with tamoxifen.) This metabolite undergoes multiple oxidations including 4-hydroxylation by CYP2D6 to endoxifen. Tamoxifen 4-hydroxylation via multiple CYPs to 4-hydroxytamoxifen represents a minor primary metabolic route of tamoxifen. A small portion of endoxifen plasma concentrations appears to result from CYP3A-catalyzed $\mathrm{N}$-demethylation of 4-hydroxytamoxifen. The hydroxylated metabolites undergo conjugation by phase II enzymes (eg, sulfotransferases [SULTs]). UGT, Uridine diphosphate-glucuronosyltransferase.

which codes for a fully functional enzyme. $C Y P 2 D 6^{*} 2$, *33, and *35 alleles contain point mutations that do not affect the catalytic properties of the gene product. Alleles associated with no enzymatic activity (CYP2D6 $\left.3-{ }^{*} 8,{ }^{*} 11-{ }^{*} 16,{ }^{*} 18-{ }^{*} 20,{ }^{*} 38,{ }^{*} 40,{ }^{*} 42,{ }^{*} 44\right)$ or reduced activity (CYP2D6 $9,{ }^{*} 10,{ }^{*} 17,{ }^{*} 29,{ }^{*} 36,{ }^{*} 37$, *41) have been identified. ${ }^{13-16}$ The presence of multiple copies of CYP2D6 alleles (ie, * $1,{ }^{*} 2,{ }^{*} 35$, and *41) has been reported in subjects with unusually high CYP2D6 catalytic activity. ${ }^{17,18}$

In our pilot clinical study we have established the link between endoxifen plasma concentrations and CYP2D6 status. ${ }^{7}$ More recently, we tested the association between tamoxifen metabolism and 4 CYP2D6 null alleles and concomitantly administered CYP2D6 inhibitors in 80 breast cancer patients treated with 20 $\mathrm{mg} / \mathrm{d}$ tamoxifen. ${ }^{10}$ These data indicate that intersubject variability in the endoxifen concentration is accounted for in part by CYP2D6 genotype and by concomitant medications that inhibit CYP2D6 activity. However, 
we observed a large interpatient variability in the endoxifen concentration even after correcting for CYP2D6 status. This residual variability could result in part from rare $C Y P 2 D 6$ null alleles or variants that are associated with reduced activity and that were not determined in our previous study. To address this issue, we have carried out a thorough investigation of the CYP2D6 genetic polymorphisms in the whole cohort of 158 patients. In addition to the 4 alleles studied previously, 29 additional alleles with different effects on CYP2D6 activity were analyzed by use of the researchbased AmpliChip CYP450 Test (Roche Molecular Systems, Alameda, Calif). In addition, we intended to replicate our initial observation that concomitantly prescribed drugs which are known to be CYP2D6 inhibitors reduce endoxifen plasma concentrations.

\section{METHODS \\ Patients}

Eligible women were recruited into a prospective cohort registry from 3 breast cancer clinics - the Lombardi Comprehensive Cancer Center at Georgetown University Medical Center, Washington, DC; the Breast Oncology Program at the University of Michigan Comprehensive Cancer Center, Ann Arbor, Mich; and the Indiana University Cancer Center, Indianapolis, Ind. Premenopausal and postmenopausal women (aged $\geq 18$ years) with newly diagnosed breast cancer who were starting tamoxifen as standard adjuvant therapy were included in this registry. Patients were enrolled after they had completed all primary surgery, radiation, and adjuvant chemotherapy. They were excluded from the registry if they had started tamoxifen therapy concurrently with either adjuvant chemotherapy or adjuvant radiation therapy (or both) or if they were undergoing other adjuvant endocrine therapies. Other reasons for exclusion included current long-term corticosteroid therapy (previous use during adjuvant chemotherapy was permitted) and use of clonidine, combinations of ergotamine and phenobarbital, or megestrol acetate (INN, megestrol) for hot flash therapy. Patients who were pregnant or lactating were also excluded from the registry. Enrolled patients were allowed to take vitamin E, selective serotonin reuptake inhibitors (SSRIs), or herbal remedies, provided that they had been taking these drugs for at least 4 weeks and intended to continue taking them for at least the first month while participating in the study. Likewise, patients were allowed to begin therapy with the mentioned medications while participating in the study, provided that they were willing to continue the treatment for at least 1 month. The registry protocol was approved by the institutional review boards of all 3 participating sites. All patients provided written informed consent before entry.

\section{Study design}

In this report we present data that relate to genetic polymorphisms in CYP2D6 and plasma concentrations of tamoxifen and its metabolites from 158 women who had been entered into the registry. These women were selected for this study because they had completed the necessary physical and laboratory examinations at baseline and 1, 4, 8, and 12 months after the start of tamoxifen therapy $(20 \mathrm{mg} / \mathrm{d}$ orally in a single dose in the morning) to be included in this analysis. At the mentioned time points, medical histories, including a comprehensive list of current medications, were obtained, and blood samples $(5 \mathrm{~mL})$ were drawn in most patients immediately before the following dose of tamoxifen. In some cases blood samples were drawn at random. However, given the long half-life of tamoxifen (5-7 days), ${ }^{19,20}$ we do not expect a large variability in plasma concentrations of tamoxifen or its metabolites at steady state as a result of differences in sampling time. Blood was collected in heparinized tubes, and plasma was separated within 1 hour of blood collection by centrifugation at $2060 \mathrm{~g}$. All samples (plasma and whole blood) were transferred to cryogenic vials (Corning, Cambridge, Mass), shipped to the laboratory of the Division of Clinical Pharmacology, Indiana University, on dry ice, and stored at $-80^{\circ} \mathrm{C}$ pending analysis.

\section{Sample analysis}

Analysis of concentrations of tamoxifen and its metabolites in plasma. The plasma concentrations of tamoxifen and its metabolites were determined by use of an HPLC system developed ${ }^{21}$ and subsequently modified by our group. ${ }^{3}$ This method involves a column-switching and online photocyclization technique in which the eluent, after chromatographic separation, passes through an ICT Beam Boost postcolumn photoreactor supplied with a 5-m reaction coil and a 254-nm ultraviolet lamp (Advanced Separation Technologies, Whippany, NJ), in which the photoreaction converts tamoxifen and its metabolites to highly fluorescent phenanthrene derivatives.

CYP2D6 genotyping. Genomic deoxyribonucleic acid (DNA) was extracted from the leukocyte portion of whole blood by use of a QIAamp DNA Blood Mini Kit (Qiagen, Valencia, Calif) and used for genotyping of $C Y P 2 D 6$ variants. $C Y P 2 D 6 * 3, * 4, * 6$, *7, and $* 8$ variant alleles were genotyped by use of a Taqman Allelic Discrimination Assay (Applied Biosystems, Foster City, Calif) according to the manufacturer's in- 
structions. $C Y P 2 D 6 * 10$ and $* 17$ were assayed by endonuclease-specific mutation analysis of a 4.7kilobase pair DNA fragment that contained the $C Y P 2 D 6$ gene. This DNA fragment was amplified from the genomic DNA by use of an expanded long-template polymerase chain reaction and then used as a template to determine specific genetic variants by restriction fragment length polymorphism (RFLP) analysis as described elsewhere. ${ }^{22,23}$ The digested polymerase chain reaction products were then analyzed with an Agilent 2100 Bioanalyzer (Agilent Technologies, Rockville, Md). Some samples were also tested for $C Y P 2 D 6^{*} 3$, $* 4$, *6, and *8 variants by use of the RFLP assay described previously. We also assayed for the $C Y P 2 D 6 * 41$ intronic variant recently described by Raimundo et al. ${ }^{24}$ In addition, we used the AmpliChip CYP450 Test to test for 33 CYP2D6 alleles (ie, *1 to $* 10 A B, * 11, * 14 A, * 14 B, * 15, * 17, * 19, * 20, * 25, * 26$, $* 29$ to $* 31, * 35, * 36, * 40, * 41, * 1 x N, * 2 x N, * 4 x N$, $* 10 x N, * 17 x N, * 35 x N$, and $* 41 x N$ ) in 129 samples. The AmpliChip CYP450 Test microarray contains more than 15,000 different oligonucleotide probes by which to analyze both sense and antisense strands of an amplified target DNA sample. ${ }^{25}$

\section{Statistical analysis}

The phenotype data, including endoxifen plasma concentrations and endoxifen/NDM and NDM/endoxifen plasma concentration ratios, were analyzed by use of normit plots (or quantile-quantile [Q-Q] plots) to obtain initial information about the distribution of the data. Because the normit plot itself cannot be directly used to decide how many mixture components the distribution contains, we used a mixture normal model approach, ${ }^{26-28}$ which allowed us to select the number of components using the Bayesian information criterion. ${ }^{29}$ A unique feature of the mixture model is that it assigns each sample the probability of belonging to each normal distribution.

The comparisons of endoxifen/NDM plasma concentration ratios and endoxifen plasma concentrations between genotype groups and between patients taking CYP2D6 inhibitors and those not taking CYP2D6 inhibitors were performed by use of unpaired $t$ tests. Phenotype expression in each defined genotype group was reported as mean $\pm \mathrm{SD}$. The association between CYP2D6 genotype and phenotype groups was evaluated by use of the Mantel-Haenszel chi-square test. The effect of concomitant CYP2D6 inhibitors on endoxifen plasma concentrations in different CYP2D6 genotype groups was analyzed by multiple linear regression. $P<$ .05 was considered statistically significant.

\section{RESULTS \\ Demographics}

The cohort was composed of 158 patients. The median age was 54 years (range, 30-87 years), and the mean body mass index was $28 \mathrm{~kg} / \mathrm{m}^{2}$ (range, 19-58 $\mathrm{kg} / \mathrm{m}^{2}$ ). Most of the patients were white $(91.1 \%)$, with a small representation of other ethnic groups as follows: black, 5.7\%; Arabic, 1.3\%; and Hispanic, 0.6\%. Ethnicity information could not be obtained in 2 patients $(1.3 \%)$

\section{CYP2D6 genotyping}

We performed an exhaustive genetic analysis of the CYP2D6 gene in breast cancer patients treated with tamoxifen. We screened for 33 different alleles, including multiple copies of the gene, gene deletion, and alleles that occur at low frequencies in white persons. In most patients the presence of frequent alleles (eg, CYP2D6*3, *4, *6, *7, *8, *10, and *41) was confirmed by 2 or 3 different genotyping methods (ie, RFLP, Taqman Allelic Discrimination Assay, and AmpliChip CYP450 Test). The no-call rate for the AmpliChip CYP450 Test was $0.7 \%$. The discordance between our assays and the AmpliChip CYP450 Test was less than $2 \%(2 / 129)$. In the case of the 2 discordant samples, we decided to include the results obtained by the AmpliChip CYP450 Test.

The frequencies of individual CYP2D6 genotypes are presented in Table I. Null alleles $(C Y P 2 D 6 * 3, * 4$, $* 5$, and $* 6$ ), dysfunctional alleles (CYP2D6*9, *10, $* 17, * 29$, and $* 41)$, and functional alleles (CYP2D6*1, $* 2$, and *35) were designated as poor metabolizer (PM), intermediate metabolizer (IM), and extensive metabolizer (EM) alleles, respectively. ${ }^{15}$ Multiple copies of any functional allele were designated as ultrarapid metabolizer (UM). ${ }^{15}$ As expected, the most frequent alleles were $* 1(0.453), * 4(0.161)$, and $* 2(0.13)$. CYP2D6*4 was the most common null allele and the only allele present in PM/PM genotype patients. The frequencies of other null alleles were $0.022(* 5), 0.013$ $(* 3)$, and $0.003(* 6)$. The IM genotype groups (ie, $\mathrm{IM} / \mathrm{PM}$ and IM/IM) represented $7.6 \%$, rising to $34.2 \%$ if EM/PMs were included. CYP2D6*41 was the most frequent dysfunctional allele $(0.089)$, followed by CYP2D6*10 (0.035) and *9 (0.009). Of the patients, $38 \%$ had an EM/EM genotype, most of whom $(55 \%)$ were homozygotes for $C Y P 2 D 6 * 1$. The remaining $45 \%$ were different combinations of $C Y P 2 D 6 * 1$, *2, and *35. The UM/EM group represented $4.4 \%$ of the patients and was primarily composed of multiple copies of the wild-type allele $\left(C Y P 2 D 6^{*} 1 x n\right)$. With regard to ethnicity, the black patients carried the $C Y P 2 D 6 * 1 / * 1$ 
$(\mathrm{n}=5), * 1 / * 29(\mathrm{n}=1), * 1 / * 5(\mathrm{n}=1), * 17 / * 41(\mathrm{n}=1)$, or $* 10 / * 4(\mathrm{n}=1)$ genotype. The only Hispanic patient in our study, the 2 Arabic patients, and the 2 patients whose ethnicity was unknown had the CYP2D $6 * 1 / * 4$, $* 1 / * 1$ and $* 1 / * 10$, and $* 1 / * 4$ and $* 10 / * 35$ genotypes, respectively. All of the other genotypes were found in white patients. In our study we did not find any individual carrying the low-frequency alleles (CYP2D6*7, $* 8, * 11, * 14, * 15, * 18, * 19, * 20, * 25, * 26, * 30, * 31$, $* 36$, or $* 40$ ).

\section{Plasma concentrations of tamoxifen and its metabolites}

Our previous studies have indicated that steady-state plasma concentrations of tamoxifen and its metabolites are achieved in 4 months. ${ }^{10}$ The plasma concentrations at 4, 8, and 12 months were measured in selected patients and found to be comparable. Therefore we present the data collected after 4 months of treatment with tamoxifen. At the fourth month, 46 patients (29\%) were receiving CYP2D6 inhibitors concomitantly, 33 of whom were taking SSRIs. We were not able to obtain information about concomitant medications in 18 patients. These 18 patients were excluded from the analysis of the effect of CYP2D6 inhibitors on tamoxifen pharmacokinetics.

Mean plasma concentrations $( \pm S D)$ of tamoxifen, NDM, 4-hydroxytamoxifen, and endoxifen in the whole cohort $(\mathrm{N}=158)$ were $334.5 \pm 147.9 \mathrm{nmol} / \mathrm{L}$, $695.2 \pm 353.8 \mathrm{nmol} / \mathrm{L}, 7.4 \pm 3.7 \mathrm{nmol} / \mathrm{L}$, and $61.2 \pm$ $40.6 \mathrm{nmol} / \mathrm{L}$, respectively. There was no significant difference in mean plasma concentrations of tamoxifen, NDM, and 4-hydroxytamoxifen between patients receiving concomitant CYP2D6 inhibitors and those not receiving concomitant CYP2D6 inhibitors (332.2 \pm $151.1 \mathrm{nmol} / \mathrm{L}$ versus $337.6 \pm 156.7 \mathrm{nmol} / \mathrm{L}[P=.85]$, $686.9 \pm 328.2 \mathrm{nmol} / \mathrm{L}$ versus $638.9 \pm 326.7 \mathrm{nmol} / \mathrm{L}[P$ $=.42]$, and $7.02 \pm 3.6 \mathrm{nmol} / \mathrm{L}$ versus $7.8 \pm 3.9 \mathrm{nmol} / \mathrm{L}$ $[P=.29]$, respectively). However, the mean endoxifen plasma concentration was significantly lower in patients taking CYP2D6 inhibitors than in those not taking any concomitant CYP2D6 inhibitors (39.6 \pm 28.4 $\mathrm{nmol} / \mathrm{L}$ versus $71.5 \pm 41.2 \mathrm{nmol} / \mathrm{L}, P<.01)$. These findings reflect the importance of the CYP2D6 enzyme in the formation of endoxifen.

It has been shown that the plasma concentration of CYP2D6 substrates follows a multimodal distribution. ${ }^{13}$ To evaluate this phenomenon in our study, we used the normit plot (or Q-Q plot) and a mixture model approach, as described in the "Statistical Analysis" section. When all patients were included in the analysis, the endoxifen plasma concentration and NDM/endox-
Table I. CYP2D6 genotype frequencies in whole cohort of breast cancer patients $(\mathrm{N}=158)$

\begin{tabular}{|c|c|c|}
\hline CYP2D6 genotype group & CYP2D6 genotype & $n(\%)$ \\
\hline $\mathrm{PM} / \mathrm{PM}(4.4 \%)$ & $* 4 / * 4$ & $7(4.4)$ \\
\hline \multirow[t]{5}{*}{$\mathrm{IM} / \mathrm{PM}(3.8 \%)$} & $* 41 / * 4$ & $2(1.2)$ \\
\hline & $* 10 / * 4$ & $1(0.6)$ \\
\hline & $* 10 / * 4 x n$ & $1(0.6)$ \\
\hline & $* 41 / * 3$ & $1(0.6)$ \\
\hline & $* 9 / * 5$ & $1(0.6)$ \\
\hline \multirow[t]{5}{*}{$\mathrm{IM} / \mathrm{IM}(3.8 \%)$} & $* 41 / * 41 x n$ & $2(1.2)$ \\
\hline & $* 9 / * 41$ & $1(0.6)$ \\
\hline & $* 10 / * 41$ & $1(0.6)$ \\
\hline & $* 17 / * 41$ & $1(0.6)$ \\
\hline & $* 41 / * 41$ & $1(0.6)$ \\
\hline \multirow[t]{7}{*}{ EM/PM (26.6\%) } & $* 1 / * 4$ & $25(15.8)$ \\
\hline & $* 1 / * 5$ & $5(3.1)$ \\
\hline & $* 2 / * 4$ & $5(3.1)$ \\
\hline & $* 1 / * 3$ & $3(1.8)$ \\
\hline & $* 35 / * 4$ & $2(1.2)$ \\
\hline & $* 1 / * 6$ & $1(0.6)$ \\
\hline & $* 35 / * 5$ & $1(0.6)$ \\
\hline \multirow{8}{*}{ EM/IM (17.7\%) } & $* 1 / * 41$ & $15(9.5)$ \\
\hline & $* 2 / * 10$ & $4(2.5)$ \\
\hline & $* 1 / * 10$ & $3(1.8)$ \\
\hline & $* 35 / * 41$ & $2(1.2)$ \\
\hline & $* 1 / * 29$ & $1(0.6)$ \\
\hline & $* 2 / * 9$ & $1(0.6)$ \\
\hline & $* 2 / * 41$ & $1(0.6)$ \\
\hline & $* 35 / * 10$ & $1(0.6)$ \\
\hline EM/IMxn $(0.6 \%)$ & $* 2 / * 41 x n$ & $1(0.6)$ \\
\hline \multirow[t]{5}{*}{ EM/EM $(38 \%)$} & $* 1 / * 1$ & $33(20.9)$ \\
\hline & $* 1 / * 2$ & $14(8.9)$ \\
\hline & $* 1 / * 35$ & $6(3.8)$ \\
\hline & $* 2 / * 2$ & $5(3.1)$ \\
\hline & $* 2 / * 35$ & $2(1.2)$ \\
\hline $\mathrm{UM} / \mathrm{PM}(0.6 \%)$ & $* 2 x n / * 4$ & $1(0.6)$ \\
\hline \multirow[t]{3}{*}{$\mathrm{UM} / \mathrm{EM}(4.4 \%)$} & $* 1 / * 1 x n$ & $3(1.8)$ \\
\hline & $* 1 x n / * 2$ & $3(1.8)$ \\
\hline & $* 1 / * 2 x n$ & $1(0.6)$ \\
\hline
\end{tabular}

PM, CYP2D6 null allele; IM, CYP2D6 dysfunctional allele; EM, $C Y P 2 D 6$ functional allele; IMxn, 2 or more $C Y P 2 D 6$ dysfunctional alleles; UM, 2 or more $C Y P 2 D 6$ functional alleles.

ifen and endoxifen/NDM plasma concentration ratios showed curved normit plots (Fig 2, A), indicating that the distribution of all 3 parameters was not homogeneous within the study population but, rather, was composed of a mixture of normal distributions. When these variables were fitted into the mixture normal model, the endoxifen plasma concentration contained 2 components and both NDM/endoxifen and endoxifen/NDM plasma concentration ratios contained 4 components (Fig 2, B). According to the mixture model analysis, each patient was assigned a probability of belonging to 

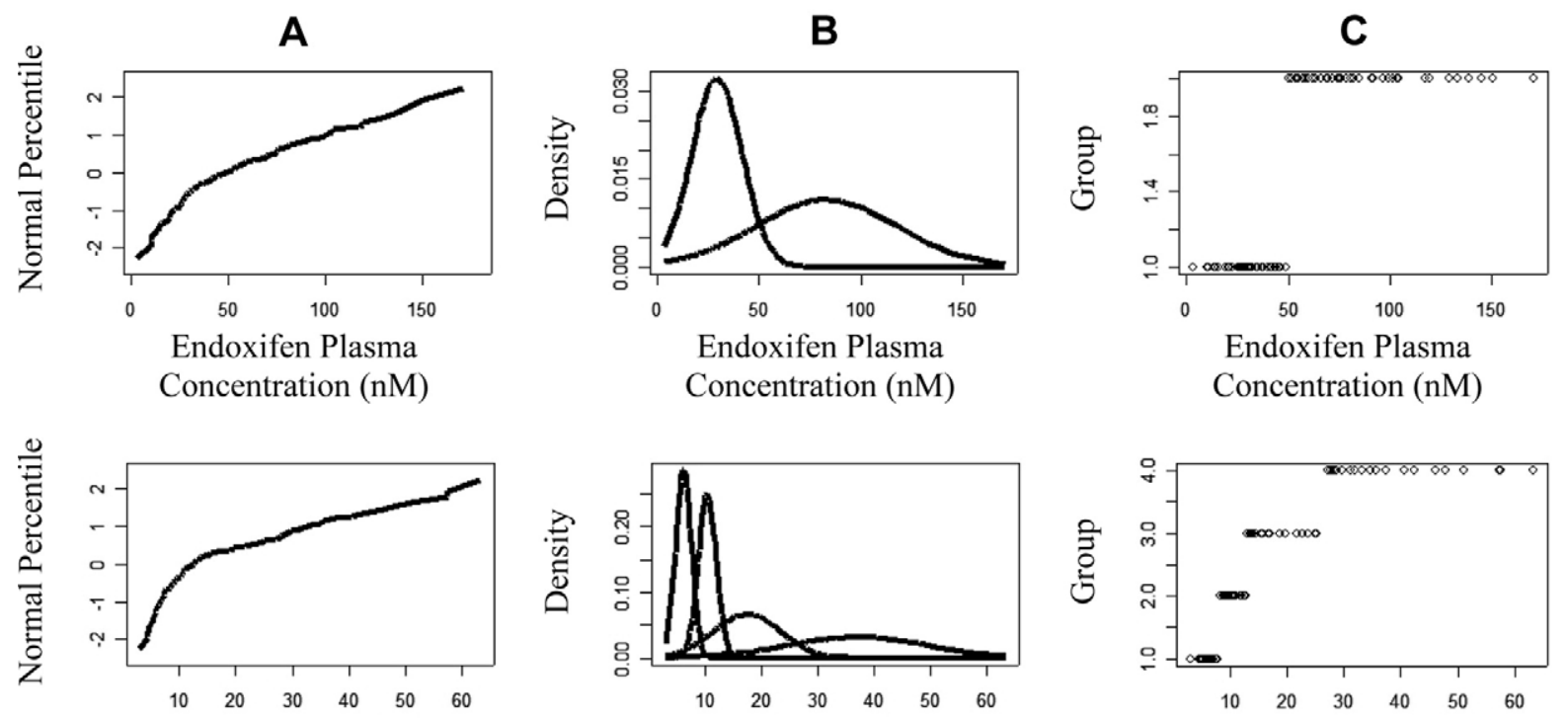

NDM/Endoxifen Plasma Ratio

NDM/Endoxifen Plasma Ratio

NDM/Endoxifen Plasma Ratio
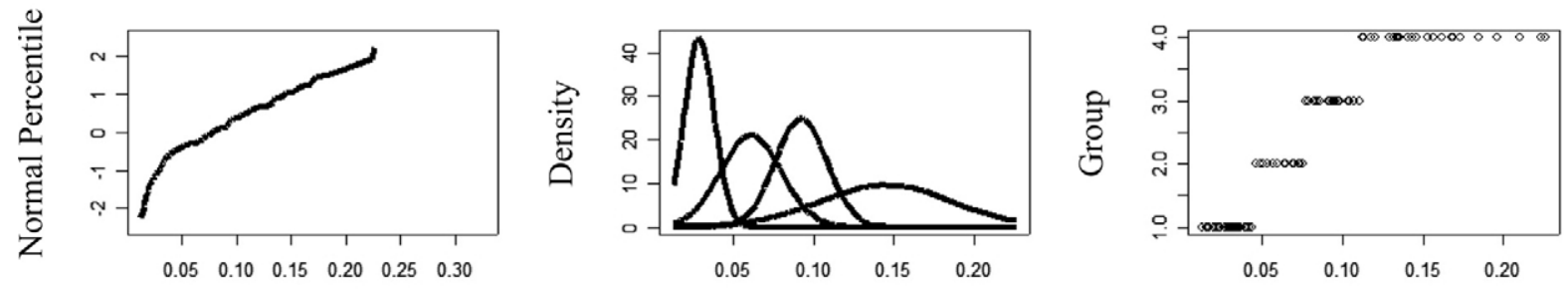

Endoxifen/NDM Plasma Ratio

Endoxifen/NDM Plasma Ratio

Endoxifen/NDM Plasma Ratio

Fig 2. Distribution of plasma concentrations of endoxifen (upper panels), as well as plasma concentration ratios of NDM/endoxifen (middle panels) and endoxifen/ $N$-desmethyltamoxifen (NDM) (lower panels), in whole cohort of breast cancer patients $(\mathrm{N}=158)$ after 4 months of treatment with tamoxifen $(20 \mathrm{mg} / \mathrm{d})$. A . Normit plots. The jagged appearance of the line indicates that the population is composed of more than 1 group. $\mathbf{B}$, Mixture normal model showing the number of groups contained in the population. The number of groups is determined with the Bayesian information criterion. The cut points are $52.2 \mathrm{nmol} / \mathrm{L}$ for endoxifen concentration; 7.4, 11.8, and 22.7 for NDM/endoxifen concentration ratio; and $0.05,0.09$, and 0.16 for endoxifen/NDM concentration ratio. $\mathbf{C}$, Classification of the population based on every sample's probability of belonging to each group. For example, if a patient's endoxifen concentration is below the cut point of $52.2 \mathrm{nmol} / \mathrm{L}$ (upper panel, B), it is more probable that this patient belongs to the first mixture component.

each group. For example, if a patient's endoxifen plasma concentration was below the cut point of 52.2 $\mathrm{nmol} / \mathrm{L}$, it was more probable that this patient belonged to the first normal component; otherwise, it was more likely that the patient was part of the second component. On the basis of these probabilities, the patients were clustered into different groups or classes (Fig 2, $C)$. Accordingly, the endoxifen plasma concentration identified 2 distinct subgroups of patients within the population, whereas NDM/endoxifen and endoxifen/
NDM plasma concentration ratios identified 4, suggesting that the ratios were more efficient in discriminating phenotypic subpopulations. The same pattern of distribution of the endoxifen plasma concentration and NDM/endoxifen and endoxifen/NDM plasma concentration ratios was observed when patients who were taking CYP2D6 inhibitors along with tamoxifen were excluded from the analysis, further supporting the existence of the mentioned subgroups within our study population (Fig 3). NDM/endoxifen and endoxifen/ 

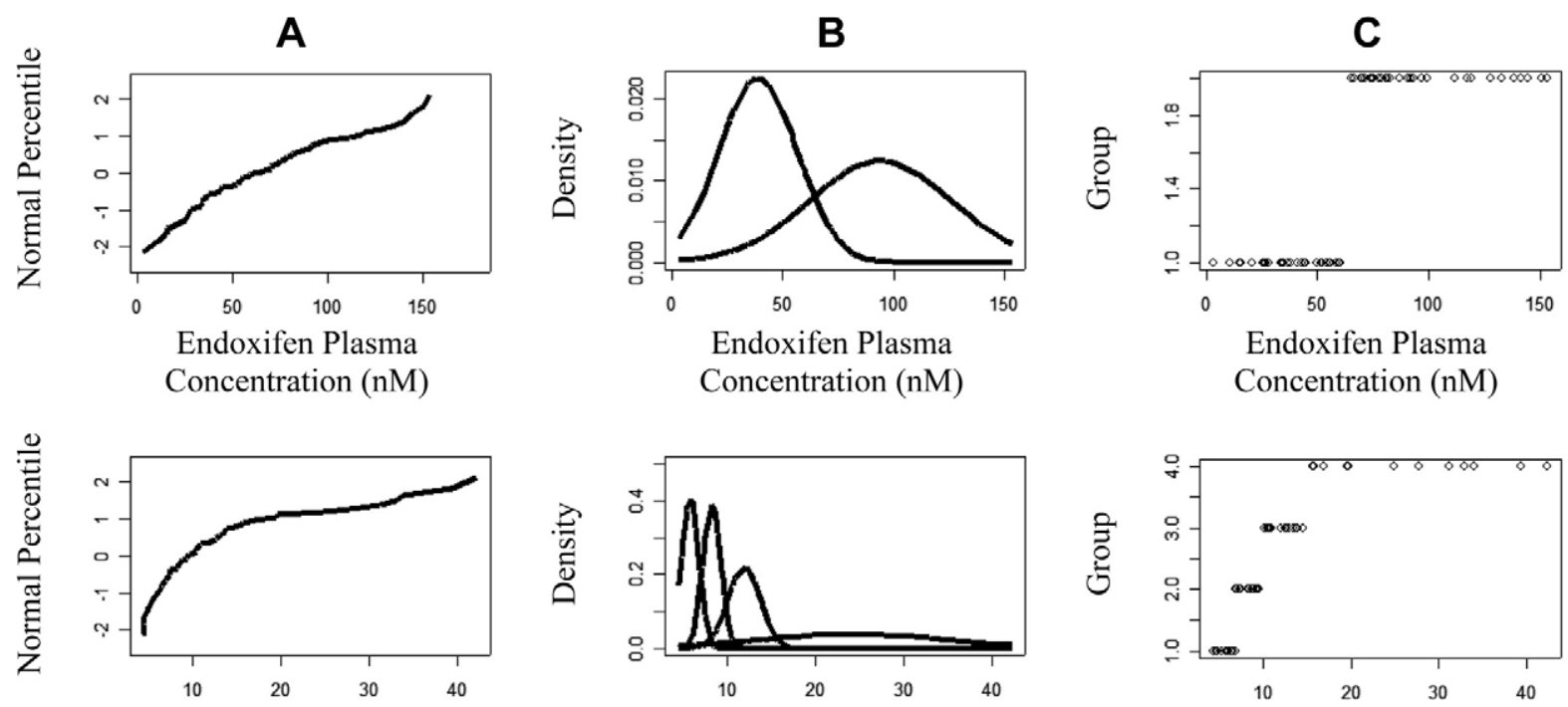

NDM/Endoxifen Plasma Ratio

NDM/Endoxifen Plasma Ratio

NDM/Endoxifen Plasma Ratio
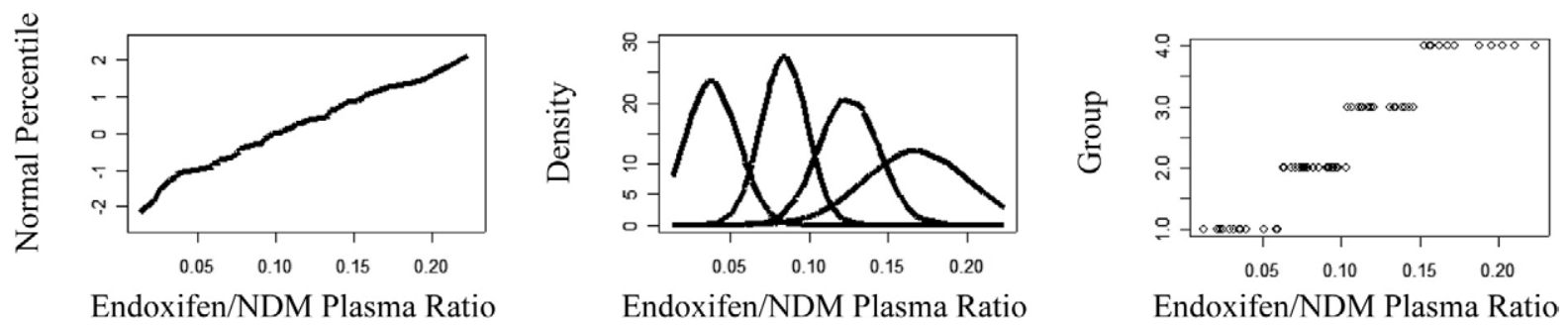

Fig 3. Distribution of plasma concentrations of endoxifen (upper panels), as well as plasma concentration ratios of NDM/endoxifen (middle panels) and endoxifen/NDM (lower panels), in breast cancer patients $(\mathrm{n}=94)$ after 4 months of treatment with tamoxifen $(20 \mathrm{mg} / \mathrm{d})$ and no concomitant CYP2D6 inhibitors. A, Normit plots. The jagged appearance of the line indicates that the population is composed of more than 1 group. B, Mixture normal model showing the number of groups contained in the population. The number of groups is determined with the Bayesian information criterion. The cut points are $60.5 \mathrm{nmol} / \mathrm{L}$ for endoxifen concentration; 5.9, 8.9, and 13.9 for NDM/endoxifen concentration ratio; and $0.06,0.11$, and 0.17 for endoxifen/NDM concentration ratio. C, Classification of the population based on every sample's probability of belonging to each group. For example, if a patient's endoxifen concentration is below the cut point of $60.5 \mathrm{nmol} / \mathrm{L}$ (upper panel, B), it is more probable that this patient belongs to the first mixture component.

NDM plasma concentration ratios provided the same information. For the sake of space and clarity, we decided to use the endoxifen/NDM plasma concentration ratio alone in subsequent analyses.

\section{Associations of CYP2D6 genotypes with endoxifen plasma concentration and with endoxifen/NDM concentration ratio}

The associations of the endoxifen/NDM plasma concentration ratio and the plasma concentration of endoxifen with $C Y P 2 D 6$ genotypes are shown in Fig 4, $A$ and
$B$, respectively. Three distinct genotype groups could be identified in the distribution of endoxifen/NDM plasma concentration ratio as follows: 1 group with low ratios (mean, $0.04 \pm 0.02$ ) represented by patients lacking any fully functional $C Y P 2 D 6$ allele (triangles in Fig 4, A), 1 group with intermediate ratios (mean, 0.08 \pm 0.045 ) composed of patients carrying only 1 fully functional CYP2D6 allele (circles in Fig 4, A), and a third group with higher ratios (mean, $0.15 \pm 0.09$ ) comprising patients with 2 or more copies of any functional or dysfunctional CYP2D6 allele (diamonds in Fig 
A

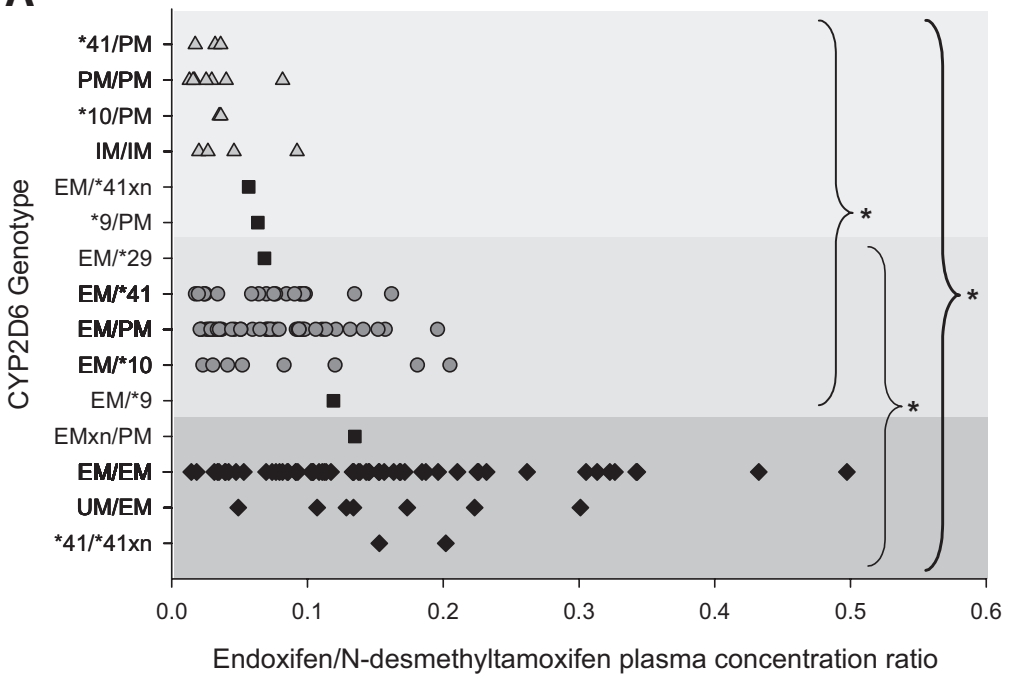

B

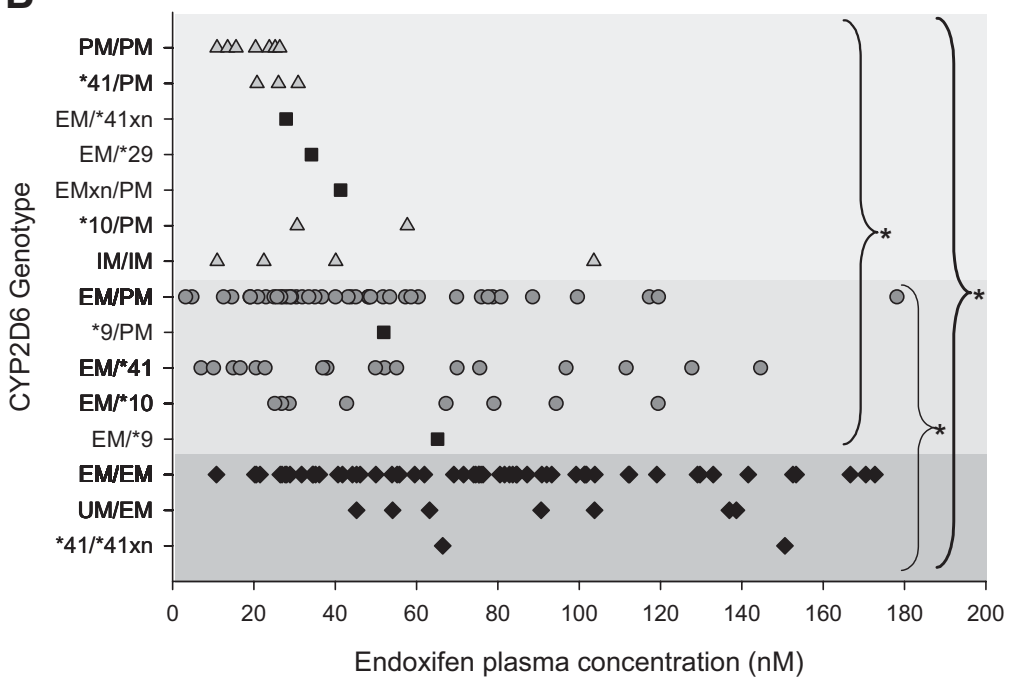

Fig 4. A, Effect of CYP2D6 genotype on endoxifen/NDM plasma concentration ratio in whole cohort of breast cancer patients $(\mathrm{N}=158)$ after 4 months of treatment with tamoxifen $(20 \mathrm{mg} / \mathrm{d})$. The genotype groups have been ranked according to their mean values, with the lowest mean at the top and the highest at the bottom. Those genotypes represented by only 1 patient were excluded from the comparison between groups. Solid symbols represent individual values. Triangles indicate patients lacking any fully functional CYP2D6 allele (mean, $0.04 \pm 0.02$ ), circles indicate patients carrying only 1 fully functional CYP2D6 allele (mean, $0.08 \pm 0.04$ ), diamonds indicate patients with 2 or more copies of any functional or dysfunctional CYP2D6 allele (mean, $0.15 \pm 0.09$ ), and squares indicate patients excluded from the group comparisons. Asterisk, $P<.001$. B, Effect of CYP2D6 genotype on endoxifen plasma concentration in whole cohort of breast cancer patients $(\mathrm{N}=158)$ after 4 months of treatment with tamoxifen $(20 \mathrm{mg} / \mathrm{d})$. The genotype groups have been ranked according to their mean values, with the lowest mean at the top and the highest at the bottom. Those genotypes represented by only 1 patient were excluded from the comparison between groups. Solid symbols represent individual values. Triangles indicate patients lacking any fully functional CYP2D6 allele (mean, $29.9 \pm 22.8 \mathrm{nmol} / \mathrm{L}$ ); circles indicate patients carrying only 1 fully functional CYP2D6 allele (mean, $51.9 \pm 36.3 \mathrm{nmol} / \mathrm{L}$ ); diamonds indicate patients with 2 or more copies of any functional or dysfunctional CYP2D6 allele (mean, $78.9 \pm 41.6 \mathrm{nmol} / \mathrm{L}$ ); squares indicate patients excluded from the group comparisons. Asterisk, $P<.01$. PM, Poor metabolizer; IM, intermediate metabolizer; EM, extensive metabolizer; UM, ultrarapid metabolizer. 
4, A). Genotypes represented by only 1 patient were excluded from the group comparison (squares in Fig 4, $A)$. The endoxifen/NDM plasma concentration ratio was significantly different between groups $(P<.001)$. Although the endoxifen plasma concentration was also associated with CYP2D6 genotypes, this association was less marked compared with that of the ratio (Fig 4, $B)$. Therefore we chose the ratio as a more sensitive marker of CYP2D6.

When the patients taking CYP2D6 inhibitors were excluded from the analysis, the separation of genotype groups by endoxifen/NDM plasma concentration ratio became more evident (Fig 5, A). Although the same applies to the endoxifen plasma concentration, its high variability reduced its ability to discriminate separate genotypic groups (Fig 5, B).

After identifying distinct phenotype and genotype subgroups within the study population, we analyzed how these groups related to each other. We calculated the relative representation of the different $C Y P 2 D 6$ genotypes in each phenotype group or class defined by the mixture normal model (Table II). Genotypes represented by only 1 patient were not considered in this analysis. The relative frequencies of the CYP2D6 genotypes followed an opposite trend to the functionality of the CYP2D6 alleles in the first phenotype group; the contrary occurred in the last phenotype group. That is, genotypes with more functional $C Y P 2 D 6$ alleles were less represented in the first phenotype group than in the last one, and vice versa. This phenomenon was observable in both the endoxifen plasma concentration and endoxifen/NDM plasma concentration ratio. It is remarkable that all of the patients with the PM/PM genotype belonged to the first group of endoxifen plasma concentration. Similarly, all of the patients lacking fully functional $C Y P 2 D 6$ alleles were present in the first 2 endoxifen/NDM plasma concentration ratio phenotype groups, and none of the patients carrying more than 2 fully functional $C Y P 2 D 6$ alleles belonged to the first endoxifen/NDM plasma concentration ratio group. These differences in the distribution of $C Y P 2 D 6$ genotype groups within each phenotype group were statistically significant $(P<.0001)$.

\section{Effect of CYP2D6 inhibitors on plasma concentrations of endoxifen}

We examined the effect of coprescribed CYP2D6 inhibitors on endoxifen plasma concentrations. There was a significant decrease in mean endoxifen plasma concentration in patients taking CYP2D6 inhibitors ( $\mathrm{n}$ $=46$ ) in comparison with those not taking any concomitant CYP2D6 inhibitors $(\mathrm{n}=94)$ within the whole cohort of 158 patients $(39.6 \pm 28.4 \mathrm{nmol} / \mathrm{L}$ versus 71.5 $\pm 41.2 \mathrm{nmol} / \mathrm{L}, P<.01)$. We divided the CYP2D6 inhibitors into 2 groups according to their inhibitory potency. ${ }^{30}$ Potent inhibitors were represented by the SSRIs paroxetine and fluoxetine $(n=19)$. Weak inhibitors consisted of sertraline and citalopram among the SSRIs $(\mathrm{n}=14)$, as well as other drugs such as celecoxib, diphenydramine, and chlorpheniramine $(\mathrm{n}=$ 13). Venlafaxine, a serotonin-norepinephrine reuptake inhibitor, does not affect CYP2D6 activity and thus was considered separately $(n=6)$. We found a more pronounced decrease in mean endoxifen plasma concentrations with potent CYP2D6 inhibitors than with weak CYP2D6 inhibitors $(24.6 \pm 16.6 \mathrm{nmol} / \mathrm{L}$ versus $50.1 \pm$ $30.4 \mathrm{nmol} / \mathrm{L}, P<.01)$. Concomitant use of venlafaxine did not show any significant effect on mean endoxifen plasma concentration $(71.7 \pm 41.3 \mathrm{nmol} / \mathrm{L}$ versus 80.8 $\pm 39.3 \mathrm{nmol} / \mathrm{L}, P=.60$ ). To separate the effect of the inhibition from that of the CYP2D6 genotype, we analyzed the effect of CYP2D6 inhibitors in those patients with the EM genotype (EM/EM) (Fig 6). As expected, the mean endoxifen plasma concentration in CYP2D6 EM patients who were not taking CYP2D6 inhibitors $(84.1 \pm 39.4 \mathrm{nmol} / \mathrm{L})$ was similar to that in patients receiving venlafaxine $(93.6 \pm 38.6 \mathrm{nmol} / \mathrm{L})(P=.72)$. There was a trend toward a decrease in mean endoxifen plasma concentration in patients taking weak CYP2D6 inhibitors $(63.9 \pm 36.9 \mathrm{nmol} / \mathrm{L})$ compared with that in patients not receiving CYP2D6 inhibitors, but this difference did not reach statistical significance $(P=.15)$. The concomitant use of potent CYP2D6 inhibitors resulted in a marked reduction in mean plasma endoxifen concentration $(23.5 \pm 9.5 \mathrm{nmol} / \mathrm{L})$ in comparison with the concentrations achieved when none of these drugs was coadministered $(P<.0001)$. This low mean endoxifen plasma concentration brought about by the potent inhibitors of CYP2D6 was comparable to that in patients with the CYP2D6 PM genotype status (19.4 \pm $6.1 \mathrm{nmol} / \mathrm{L}, P=.43)$, suggesting a "phenocopy."

We next evaluated the effect of CYP2D6 inhibitors on the endoxifen plasma concentration in different CYP2D6 genotype groups (Fig 7). The same trend described in EM/EMs was observed in other $C Y P 2 D 6$ genotypes $(P=.003)$. Weak inhibitors slightly reduced the endoxifen plasma concentration, whereas potent inhibitors consistently caused a significant decrease. This effect was not so clear in the CYP2D6 EM/*10 genotype, probably because of the small number of patients included in this category. It is worth noting that the UM/EM group is the only genotype group that appeared not to be converted into a PM status by CYP2D6 potent inhibitors. It is also remarkable that 
A

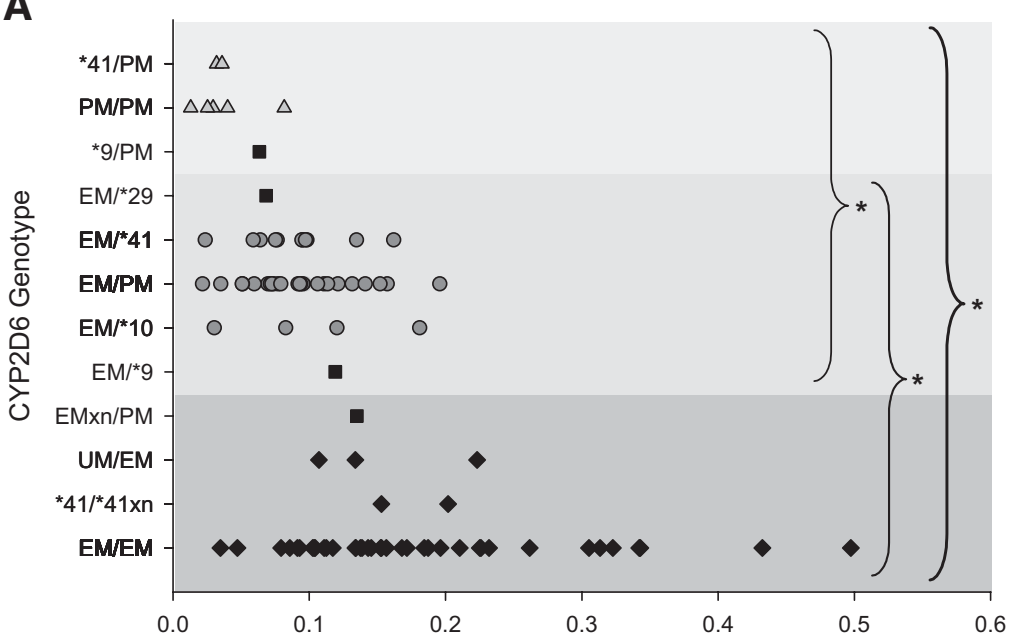

Endoxifen/N-desmethyltamoxifen plasma concentration ratio

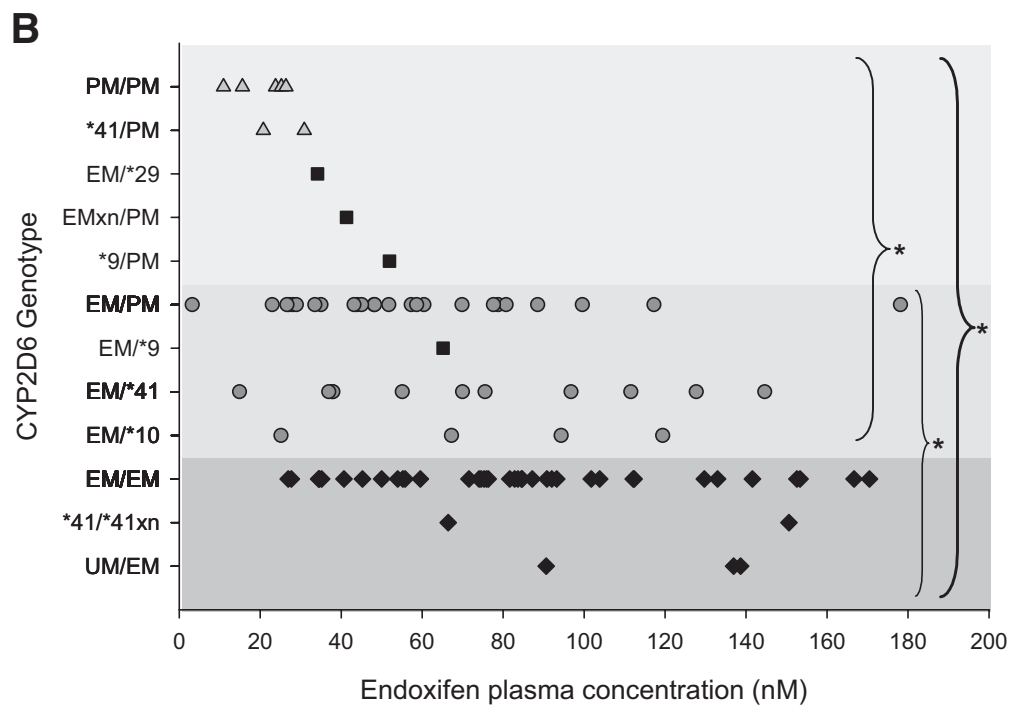

Fig 5. A, Effect of $C Y P 2 D 6$ genotype on endoxifen/NDM ratio in breast cancer patients $(\mathrm{n}=94)$ after 4 months of treatment with tamoxifen $(20 \mathrm{mg} / \mathrm{d})$ and no concomitant CYP2D6 inhibitors. The genotype groups have been ranked according to their mean values, with the lowest mean at the top and the highest at the bottom. Those genotypes represented by only 1 patient were excluded from the comparison between groups. Solid symbols represent individual values. Triangles indicate patients lacking any fully functional CYP2D6 allele (mean, $0.04 \pm 0.02$ ), circles indicate patients carrying only 1 fully functional CYP2D6 allele (mean, $0.09 \pm 0.04$ ), diamonds indicate patients with 2 or more copies of any functional or dysfunctional CYP2D6 allele (mean, $0.18 \pm 0.09$ ), and squares indicate patients excluded from the group comparisons. Asterisk, $P<.001$. B, Effect of $C Y P 2 D 6$ genotype on endoxifen concentration in breast cancer patients $(n=94)$ after 4 months of treatment with tamoxifen $(20 \mathrm{mg} / \mathrm{d})$ and no concomitant CYP2D6 inhibitors. The genotype groups have been ranked according to their mean values, with the lowest mean at the top and the highest at the bottom. Those genotypes represented by only 1 patient were excluded from the comparison between groups. Solid symbols represent individual values. Triangles indicate patients lacking any fully functional CYP2D6 allele (mean, $21.9 \pm 6.8 \mathrm{nmol} / \mathrm{L}$ ), circles indicate patients carrying only 1 fully functional CYP2D6 allele (mean, $64.2 \pm 38.2 \mathrm{nmol} / \mathrm{L}$ ), diamonds indicate patients with 2 or more copies of any functional or dysfunctional CYP2D6 allele (mean, $88.6 \pm 39.6 \mathrm{nmol} / \mathrm{L}$ ), and squares indicate patients excluded from the group comparisons. Asterisk, $P<.05$. 


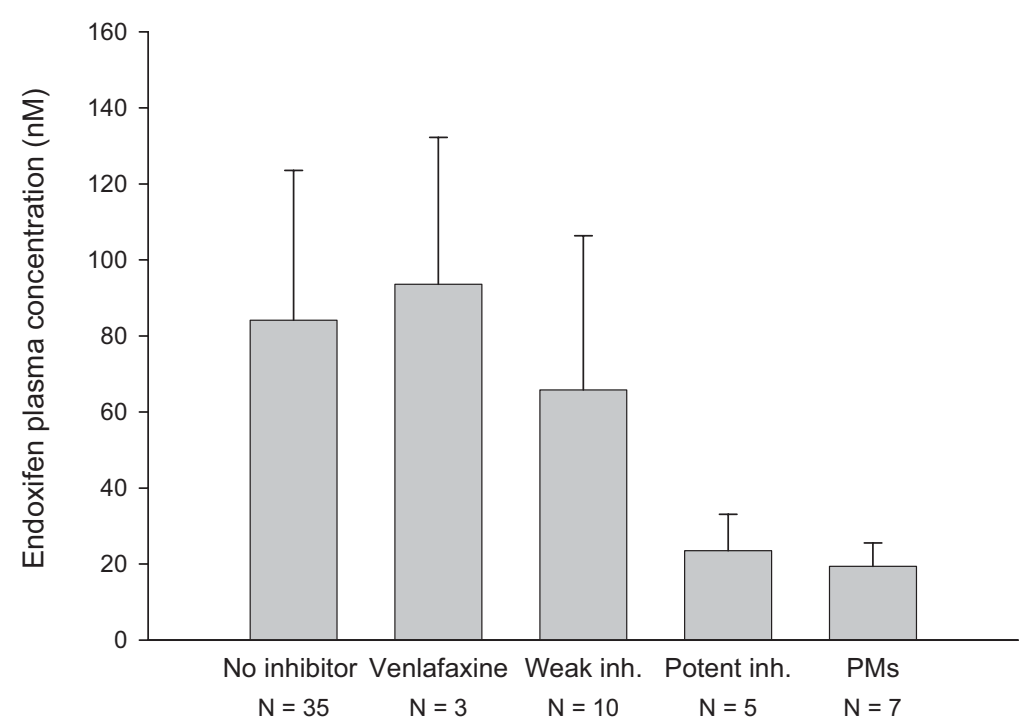

Fig 6. Effect of concomitant use of CYP2D6 inhibitors (inh) on endoxifen plasma concentration after 4 months of tamoxifen treatment $(20 \mathrm{mg} / \mathrm{d})$. Solid bars represent mean + SD. From left to right, groups are composed of EM/EMs who were receiving neither CYP2D6 inhibitors nor venlafaxine, EM/EMs who were receiving venlafaxine, EM/EMs who were receiving weak CYP2D6 inhibitors, EM/EMs who were receiving potent CYP2D6 inhibitors, and PM/PMs who were not receiving any CYP2D6 inhibitors.

Table II. Relative frequencies of CYP2D6 genotypes in each mixture model-defined phenotype group in whole cohort of breast cancer patients $(\mathrm{N}=158)$

\begin{tabular}{|c|c|c|c|c|c|c|}
\hline \multirow[b]{2}{*}{ Genotype } & \multicolumn{2}{|c|}{$\begin{array}{c}\text { Endoxifen plasma } \\
\text { concentration }\end{array}$} & \multicolumn{4}{|c|}{ Endoxifen/N-desmethyltamoxifen plasma concentration ratio } \\
\hline & Group 1 & Group 2 & Group 1 & Group 2 & Group 3 & Group 4 \\
\hline UM/EM & 0.14 & 0.86 & - & 0.14 & $0.43 *$ & $0.43 *$ \\
\hline EM/EM & $0.33 \downarrow$ & $0.67 \uparrow$ & $0.13 \downarrow$ & 0.20 & $0.33 *$ & $0.33^{*} \uparrow$ \\
\hline EM/IM & $0.54 \downarrow$ & $0.43 \uparrow$ & $0.29 \downarrow$ & $0.36^{*}$ & 0.29 & $0.07 \uparrow$ \\
\hline EM/PM & $0.67 \downarrow$ & $0.33 \uparrow$ & $0.33^{*} \downarrow$ & $0.33^{*}$ & 0.31 & $0.02 \uparrow$ \\
\hline IM/IM & $0.75 \downarrow$ & $0.25 \uparrow$ & $0.50 * \downarrow$ & $0.50 *$ & - & $-\uparrow$ \\
\hline IM/PM & $0.83 \downarrow$ & $0.17 \uparrow$ & $0.83 * \downarrow$ & 0.17 & - & $-\uparrow$ \\
\hline $\mathrm{PM} / \mathrm{PM}$ & 1.00 & - & $0.86^{*}$ & 0.14 & - & - \\
\hline
\end{tabular}

The arrrows show the trend of the relative frequency within each phenotype group.

*Highest relative frequency within each genotype group.

none of the patients with the PM/PM or *41/PM genotype were taking CYP2D6 inhibitors.

\section{DISCUSSION}

This study provides the first comprehensive analysis of the association between concentrations of the active metabolites of tamoxifen and CYP2D6 variants, as well as exposure to inhibitors of CYP2D6, providing important information that may be pragmatically applied to the individualization of tamoxifen therapy. Although the link between CYP2D6 status and tamoxifen metab- olism was established in our previous studies, the current work incorporates approaches such as normit analysis and mixture normal model distribution to quantitatively determine the association of CYP2D6 genetics and drug interactions with tamoxifen metabolism in a larger population of patients.

In this study the frequency of the PM/PM genotype was slightly lower $(4.4 \%)$ than expected $(5 \%-10 \%)$ in a white population, ${ }^{14}$ whereas the frequencies of the intermediate genotypes (ie, IM/PM and IM/IM) and the IM allele $* 41$ were similar to those in previous re- 


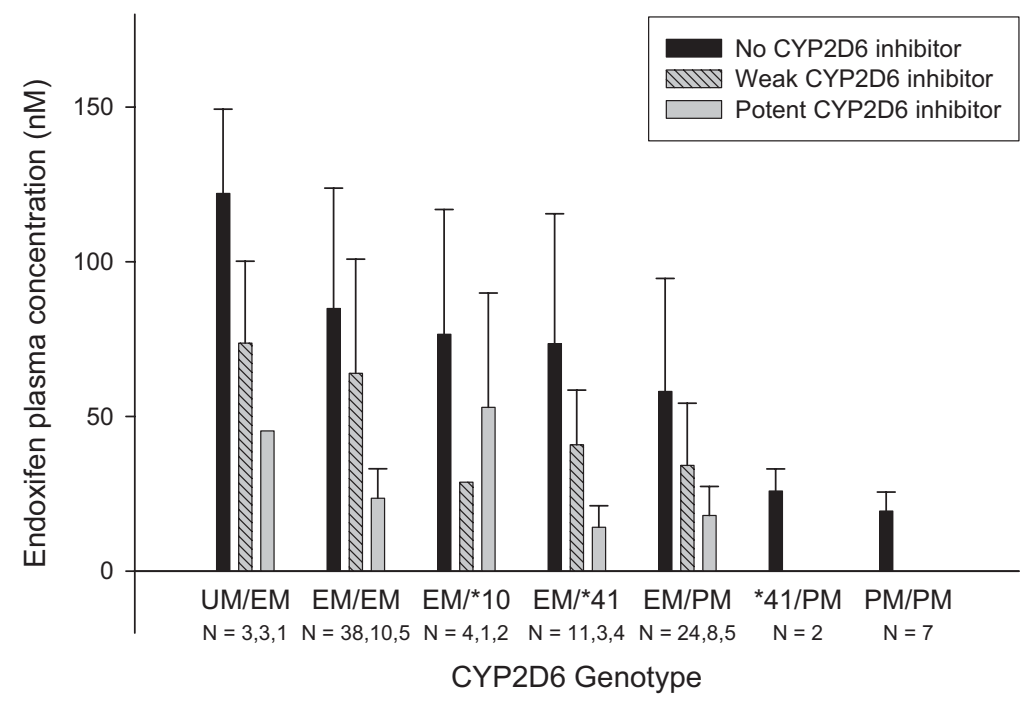

Fig 7. Effect of CYP2D6 genotype and concomitant use of CYP2D6 inhibitors on plasma endoxifen concentration after 4 months of tamoxifen treatment. Data are presented as mean + SD. Black bars, Group means for patients who were not receiving any CYP2D6 inhibitors; striped bars, group means for patients who were taking weak CYP2D6 inhibitors; gray bars, group means for patients who were taking potent CYP2D6 inhibitors.

ports. ${ }^{24}$ However, CYP2D6*10 was more common in our sample $(0.035)$ than in other comparable populations (0.015-0.018). ${ }^{14,24}$ Another difference between our study patients and other white groups was the higher representation of the UM/EM genotype (4.4\% versus $1.2 \%-1.3 \%),{ }^{14}$ which was mostly explained by multiple copies of the $* l$ allele. These discrepancies in the CYP2D6 allelic frequency may be the result of minor differences between different white populations and the small contribution of other ethnic groups to our cohort.

We examined the genotype-to-phenotype association by a combination of the mixture normal model analysis and stratification of genotype groups. The distribution of the endoxifen plasma concentration and the endoxifen/NDM plasma ratio showed 2 and 4 phenotype groups, respectively, suggesting that the ratio is a better index measure of CYP2D6 activity. On the other hand, the genotype stratification identified 3 distinct genotype groups in relation to both the endoxifen plasma concentration and its ratio to NDM. These data indicate that $C Y P 2 D 6$ genotype can explain part of the variability in the endoxifen plasma concentration and the endoxifen/NDM plasma ratio. Furthermore, when we evaluated the relative frequencies of the CYP2D6 genotypes within each endoxifen/NDM plasma concentration ratio or endoxifen plasma concentration phenotype group (Table II), CYP2D6 genotype appeared to be a good tool by which to estimate the phenotype. The pattern of the distribution of genotypes within the phenotype groups suggests that CYP2D6 genotype may allow estimation of what the endoxifen concentration would be in breast cancer patients being treated with tamoxifen in clinical settings in whom CYP2D6 genotype is known. Together, our data suggest that the iterative approaches and models used in this report appear to be valuable tools in the study of CYP2D6 and other complex genotype-phenotype relationships.

We also assessed the effect of the concomitant prescription of inhibitors of CYP2D6 on tamoxifen metabolism, which may have important clinical implications. We focused on antidepressants in this study because of their frequent use with tamoxifen for the treatment of hot flashes or mood disorders. SSRIs/ serotonin-norepinephrine reuptake inhibitors are the most promising nonhormonal treatment for hot flashes in women with breast cancer. Preliminary data support the use of citalopram and sertraline for the treatment of this frequent menopausal symptom ${ }^{31,32}$; paroxetine, fluoxetine, and venlafaxine have been reported to decrease hot flash scores by $64.6 \%, 50 \%$, and $61 \%$, respectively. ${ }^{33-35}$ In our study the simultaneous use of venlafaxine and tamoxifen did not appear to affect the endoxifen plasma concentration, whereas weak CYP2D6 inhibitors (eg, citalopram and sertraline) slightly decreased mean plasma concentrations of en- 
doxifen. In accordance with our previous reports, potent CYP2D6 inhibitors (eg, paroxetine and fluoxetine) showed the largest reduction in the concentration of endoxifen, converting CYP2D6 EMs into a PM status (phenocopy). Although we do not have enough statistical power to compare UM/EMs and the other CYP2D6 genotypes, the concomitant use of potent CYP2D6 inhibitors and tamoxifen in UM/EM patients seems to produce a less pronounced decrease in mean endoxifen plasma concentration. Because paroxetine and fluoxetine are substrates of this enzyme, it is possible that the relatively high concentration of the enzyme that results from multiple copies may rapidly metabolize these inhibitors and result in an inadequate concentration of the inhibitor at the enzyme site to adequately inhibit the conversion of NDM to endoxifen. It is of note that none of the patients with the *41/PM or PM/PM genotype were receiving concomitant CYP2D6 inhibitors. The reason for this observation is unclear, but it is consistent with a previous report that patients who are PMs of CYP2D6 had a low incidence of severe hot flashes. ${ }^{11}$ Because most of the potent inhibitors are CYP2D6 substrates, the possibility that PM patients may not tolerate these drugs or PMs may not require prescription of these SSRIs cannot be ruled out.

Available in vitro and clinical evidence points toward an important role for endoxifen in the clinical effect of tamoxifen. The data from this study together with our previous reports indicate a strong association between endoxifen concentrations, CYP2D6 genotypes, and inhibitors of the enzyme. In a recent retrospective analysis of a randomized, blinded, prospective clinical study, we found that breast cancer patients who were PMs of CYP2D6 benefit less from tamoxifen therapy compared with EMs. ${ }^{11}$ However, some variability in the endoxifen plasma concentration remains unexplained even after correction by $C Y P 2 D 6$ genotype and medication history. Although the contribution of CYP3A to the endoxifen concentration appears to be very small, this route may become apparent when CYP2D6 activity is diminished. In addition, endoxifen plasma concentrations are likely to be dependent on its formation and clearance by phase II enzymes (eg, sulfation and probably glucuronidation). It follows that CYP2D6 and other factors should be considered for a full understanding of the intersubject variability of endoxifen concentrations.

In conclusion, if the preliminary associations between clinical outcomes of tamoxifen and CYP2D6 genotype are confirmed, ${ }^{11}$ analyses of endoxifen and CYP2D6 may be useful to optimize tamoxifen treat- ment. On the other hand, the endoxifen/NDM plasma ratio may serve as a marker of CYP2D6 activity during tamoxifen treatment. Although some SSRIs greatly interfere with tamoxifen metabolism, citalopram, sertraline, and venlafaxine appear to have less impact on the endoxifen concentration and thus are probably better therapeutic alternatives in breast cancer patients undergoing tamoxifen therapy who require the use of antidepressants.

Dr Flockhart is a consultant for F. Hoffmann-La Roche, Basel, Switzerland. Dr Borges received funding from the Merck Foundation from 2003 to 2005. Dr Stearns served as a consultant to Wyeth Pharmaceuticals and received research funding from GlaxoSmithKline Pharmaceuticals. None of the other authors has a conflict of interest.

\section{References}

1. Fisher B, Costantino JP, Wickerham DL, Redmond CK, Kavanah M, Cronin WM, et al. Tamoxifen for prevention of breast cancer: report of the National Surgical Adjuvant Breast and Bowel Project P-1 Study. J Natl Cancer Inst 1998;90:1371-88.

2. Osborne CK. Tamoxifen in the treatment of breast cancer. N Engl J Med 1998;339:1609-18.

3. Desta Z, Ward BA, Soukhova NV, Flockhart DA. Comprehensive evaluation of tamoxifen sequential biotransformation by the human cytochrome $\mathrm{P} 450$ system in vitro: prominent roles for CYP3A and CYP2D6. J Pharmacol Exp Ther 2004;310:1062-75.

4. Rochat B. Role of cytochrome P450 activity in the fate of anticancer agents and in drug resistance: focus on tamoxifen, paclitaxel and imatinib metabolism. Clin Pharmacokinet 2005;44:349-66.

5. Scripture CD, Sparreboom A, Figg WD. Modulation of cytochrome $\mathrm{P} 450$ activity: implications for cancer therapy. Lancet Oncol 2005;6:780-9.

6. Jordan VC, Collins MM, Rowsby L, Prestwich G. A monohydroxylated metabolite of tamoxifen with potent antioestrogenic activity. J Endocrinol 1977;75:305-16.

7. Stearns V, Johnson MD, Rae JM, Morocho A, Novielli A, Bhargava P, et al. Active tamoxifen metabolite plasma concentrations after coadministration of tamoxifen and the selective serotonin reuptake inhibitor paroxetine. J Natl Cancer Inst 2003;95:1758-64.

8. Clarke R, Liu MC, Bouker KB, Gu Z, Lee RY, Zhu Y, et al. Antiestrogen resistance in breast cancer and the role of estrogen receptor signaling. Oncogene 2003;22:7316-39.

9. Lien EA, Solheim E, Kvinnsland S, Ueland PM. Identification of 4-hydroxy-N-desmethyltamoxifen as a metabolite of tamoxifen in human bile. Cancer Res 1988;48: 2304-8.

10. Jin Y, Desta Z, Stearns V, Ward B, Ho H, Lee KH, et al. CYP2D6 genotype, antidepressant use, and tamoxifen metabolism during adjuvant breast cancer treatment. J Natl Cancer Inst 2005;97:30-9. 
11. Goetz MP, Rae JM, Suman VJ, Safgren SL, Ames MM, Visscher DW, et al. Pharmacogenetics of tamoxifen biotransformation is associated with clinical outcomes of efficacy and hot flashes. J Clin Oncol 2005;23:9312-8.

12. Hou ZY, Pickle LW, Meyer PS, Woosley RL. Salivary analysis for determination of dextromethorphan metabolic phenotype. Clin Pharmacol Ther 1991;49:410-9.

13. Dahl ML, Johansson I, Palmertz MP, Ingelman-Sundberg M, Sjoqvist F. Analysis of the CYP2D6 gene in relation to debrisoquin and desipramine hydroxylation in a Swedish population. Clin Pharmacol Ther 1992;51:12-7.

14. Sachse C, Brockmoller J, Bauer S, Roots I. Cytochrome P450 2D6 variants in a Caucasian population: allele frequencies and phenotypic consequences. Am J Hum Genet 1997;60:284-95.

15. Human Cytochrome P450 (CYP) Allele Nomenclature Committee Web site. Available from: URL:http://www. cypalleles.ki.se/cyp2d6.htm. Accessed Feb 15, 2006.

16. Zanger UM, Raimundo S, Eichelbaum M. Cytochrome P450 2D6: overview and update on pharmacology, genetics, biochemistry. Naunyn Schmiedebergs Arch Pharmacol 2004;369:23-37.

17. Lundqvist E, Johansson I, Ingelman-Sundberg M. Genetic mechanisms for duplication and multiduplication of the human CYP2D6 gene and methods for detection of duplicated CYP2D6 genes. Gene 1999;226:327-38.

18. Dahl ML, Johansson I, Bertilsson L, Ingelman-Sundberg M, Sjoqvist F. Ultrarapid hydroxylation of debrisoquine in a Swedish population. Analysis of the molecular genetic basis. J Pharmacol Exp Ther 1995;274:516-20.

19. Lonning PE, Lien EA, Lundgren S, Kvinnsland S. Clinical pharmacokinetics of endocrine agents used in advanced breast cancer. Clin Pharmacokinet 1992;22: 327-58.

20. Adam HK, Patterson JS, Kemp JV. Studies on the metabolism and pharmacokinetics of tamoxifen in normal volunteers. Cancer Treat Rep 1980;64:761-4.

21. Lee KH, Ward BA, Desta Z, Flockhart DA, Jones DR. Quantification of tamoxifen and three metabolites in plasma by high-performance liquid chromatography with fluorescence detection: application to a clinical trial. J Chromatogr B Analyt Technol Biomed Life Sci 2003; 791:245-53.

22. Desta Z, Kerbusch T, Flockhart DA. Effect of clarithromycin on the pharmacokinetics and pharmacodynamics of pimozide in healthy poor and extensive metabolizers of cytochrome P450 2D6 (CYP2D6). Clin Pharmacol Ther 1999;65:10-20.
23. Hersberger M, Marti-Jaun J, Rentsch K, Hanseler E. Rapid detection of the CYP2D6*3, CYP2D6*4, and CYP2D6*6 alleles by tetra-primer PCR and of the CYP2D6*5 allele by multiplex long PCR. Clin Chem 2000;46(Pt 1):1072-7.

24. Raimundo S, Toscano C, Klein K, Fischer J, Griese EU, Eichelbaum $\mathrm{M}$, et al. A novel intronic mutation, $2988 \mathrm{G}>\mathrm{A}$, with high predictivity for impaired function of cytochrome P450 2D6 in white subjects. Clin Pharmacol Ther 2004;76:128-38.

25. Candiotti KA, Birnbach DJ, Lubarsky DA, Nhuch F, Kamat A, Koch WH, et al. The impact of pharmacogenomics on postoperative nausea and vomiting: do CYP2D6 allele copy number and polymorphisms affect the success or failure of ondansetron prophylaxis? Anesthesiology 2005;102:543-9.

26. Schork NJ, Allison DB, Thiel B. Mixture distributions in human genetics research. Stat Methods Med Res 1996; 5:155-78.

27. McLachlan G, Peel D. Finite mixture model. New York: John Wiley \& Sons; 2000. p. 209-10.

28. Balding DJ, Bishop M, Cannings C. Handbook of statistical genetics. New York: John Wiley \& Sons; 2001. p. 746-7.

29. Schwarz G. Estimating the dimension of a model. Ann Stat 1978;6:461-4.

30. Hemeryck A, Belpaire FM. Selective serotonin reuptake inhibitors and cytochrome P-450 mediated drug-drug interactions: an update. Curr Drug Metab 2002;3:13-37.

31. Barton DL, Loprinzi CL, Novotny P, Shanafelt T, Sloan J, Wahner-Roedler D, et al. Pilot evaluation of citalopram for the relief of hot flashes. J Support Oncol 2003;1:47-51.

32. Plouffe L Jr, Trott EA, Largoza M, Hansen KA. An open trial of sertraline for menopausal hot flushes: potential involvement of serotonin in vasomotor instability. Del Med J 1997;69:481-2.

33. Stearns V, Beebe KL, Iyengar M, Dube E. Paroxetine controlled release in the treatment of menopausal hot flashes: a randomized controlled trial. JAMA 2003; 289:2827-34.

34. Loprinzi CL, Sloan JA, Perez EA, Quella SK, Stella PJ, Mailliard JA, et al. Phase III evaluation of fluoxetine for treatment of hot flashes. J Clin Oncol 2002; 20:1578-83.

35. Loprinzi CL, Kugler JW, Sloan JA, Mailliard JA, LaVasseur BI, Barton DL, et al. Venlafaxine in management of hot flashes in survivors of breast cancer: a randomised controlled trial. Lancet 2000;356:2059-63. 